\title{
Carnets
}

Revue électronique d'études françaises de l'APEF

Deuxième série - 3 | 2015

Insulaire

\section{Ile et désirs de l'interdit dans Le Rivage des Syrtes de Julien Gracq}

Ana Isabel Moniz

\section{(2) OpenEdition}

1 Journals

\section{Édition électronique}

URL : http://journals.openedition.org/carnets/1416

DOI : 10.4000/carnets. 1416

ISSN : 1646-7698

Éditeur

APEF

\section{Référence électronique}

Ana Isabel Moniz, « lle et désirs de l'interdit dans Le Rivage des Syrtes de Julien Gracq », Carnets [En ligne], Deuxième série - 3 | 2015, mis en ligne le 28 février 2015, consulté le 10 décembre 2020. URL : http://journals.openedition.org/carnets/1416 ; DOI : https://doi.org/10.4000/carnets.1416

Ce document a été généré automatiquement le 10 décembre 2020.

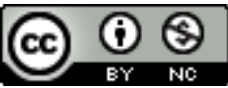

Carnets est mis à disposition selon les termes de la licence Creative Commons - Atribution - Pas d'utilisation commerciale 4.0 International. 


\title{
Ile et désirs de l'interdit dans Le Rivage des Syrtes de Julien Gracq
}

\author{
Ana Isabel Moniz
}

\section{NOTE DE L'AUTEUR}

Ce texte développe quelques questions posées dans Julien Gracq : formas sentidos e mecanismos do imaginário (2010). Lisboa : Edições Cosmos

1 Dans le parcours de l'aventure qui marque l'univers fictionnel de Julien Gracq, il est imposé aux héros une dislocation dans l'espace annoncée dès l'incipit dans bon nombre de ses romans. Soumis à une impulsion incontournable qui les fait fuir, les protagonistes vont couper les amarres avec leur passé. Temps et espace, indissociables, se présentent toujours comme alliés, chacun ayant son rôle significatif mais en même temps se complètent l'un l'autre: d'une part, le temps est à la fois lieu physique et parcelle de temps. D'autre part, l'espace équivaut à un positionnement esthétique, qu'il s'agisse de l'île, de la forêt ou de la mer. Tous ces espaces virtuels correspondent au monde imaginaire de Gracq, choisis et mis en scène pour faire évoluer les héros dans leurs aventures.

Dans Le Rivage des Syrtes, publié en $1951^{1}$ et récompensé avec le Prix Goncourt, distinction que Gracq refusera pour se protéger du milieu littéraire, la traversée de la mer des Syrtes effectuée par le protagoniste vers l'Île de Vezzano symbolise le voyage préliminaire d'une découverte.

Blasé de sa vie amoureuse, Aldo, jeune aristocrate d'Orsenna, décide de s'éloigner de cette ville assoupie. Il parvient à être muté en tant qu'observateur dans une garnison éloignée, sur la côte des Syrtes. La mission consiste à surveiller la côte car, de l'autre côté de la mer, se trouve le Farghestan, le pays ennemi depuis plus de trois cents ans. La surveillance et les longues promenades à cheval, qui ne l'éloignent pas de la monotonie, caractérisent ses journées. Cependant, sa rencontre avec Vanessa Aldobrandi et l'incursion par la mer près de la côte du Farghestan vont le conduire à éprouver la vision de l'interdit. Lors de son 
parcours, le héros se sépare de la banalité du quotidien pour, finalement, contempler « la lumière sortie de la mer » (Gracq, 1989: 745). Dans l'immensité du large, qui s'étend audelà de la ligne de l'horizon, une frontière se distinguera entre le réel et le supra-réel. La mer et l'île deviennent, ainsi, un endroit mystérieux qui sépare le héros du Farghestan, une mise en scène imaginaire d'un «non-lieu » inaccessible, mais néanmoins présent, tel le corps fascinant d'un réel irréalisé, où s'inscrit la vision fantasmatique du désir. Atteindre cette île, cet endroit du désir et de la découverte de soi, c'est la question qui se pose à Aldo dans sa quête identitaire à travers la relation symbolique qui s'établit avec l'altérité. Il décide de braver l'interdit, et là, il découvre sa vraie nature.

Julien Gracq se montre sensible au mystère de l'inexplicable, à l'attrait d'une réalité à plusieurs dimensions, y compris surnaturelles. Tout ce qui suscite la curiosité de l'homme pour une autre sphère lui apprend à entrer dans son mystère intérieur. Ce dialogue dépendra de l'espace dans lequel le personnage est mis en mouvement; il le réveille dans la contemplation de l'horizon, une frontière ténue qui semble séparer ces deux mondes et qui fascine Gracq : « ce n'est pas tellement l'en-face, c'est la lisière, la bordure d'une terre habitée, si vous voulez. Évidemment, les confins, c'est une région qui m'attire beaucoup. La côte par rapport à la mer. La frontière ici » (Gracq, $1997: 217)^{2}$.

C'est, précisément, cet espace, « la lisière, la bordure » dans sa constitution, qui occupera un lieu particulier, en suggérant la présence d'un autre monde, réservé et éloigné. Cet espace semble s'étendre vers cette ligne mystérieuse, une ligne qui séduit le héros et provoque sa quête de mondes imaginaires en s'identifiant avec « le fil invisible » (Gracq, 1995a : 217) des frontières de la surréalité.

Défini selon le point de vue de chaque individu, la notion d'horizon, en ayant comme perspective la notion phénoménologique de Michel Collot, implique la subjectivité de celui qui le perçoit (Collot, 1994 : 100), pouvant désigner tout ce qu'il observe ou ce qu'il ne voit pas, et aussi ce qu'il peut éventuellement pressentir, tel qu'on peut le vérifier dans la pratique fictionnelle de Gracq. On voit, par exemple, comment cette notion d'horizon s'impose dans Le Rivage des Syrtes, associée à la perception d'une frontière imaginaire que le personnage se propose de transgresser : « Il fixait très intentionnellement l'horizon à l'avant du navire, la voix rapide, pressé de meubler le silence (...). Il fixait l'horizon sans trop sourciller, mais une boule se contractait à sa gorge et la nervosité de ses mains le trahissait »(Gracq, 1989: 731).

7 Cette ouverture sur l'invisible se revêtira d'un sens symbolique fondamental pour la compréhension du récit vu comme une transgression de frontières: "Il me semblait qu'un voile s'était déchiré » (Gracq, 1989 : 738). Cela signifie que la frontière, ainsi conçue, ne restreint pas l'individu qui la contemple, mais elle s'ouvre à lui bien avant d'avoir la possibilité d'une nouvelle relation avec le monde. Cette ligne semble s'offrir à lui comme inaccessible, mystérieuse, interdite, mais en même temps elle l'attire, l'appelle, certaine de le voir un jour franchir sa limite : « La frontière le fascinait » (Gracq, 1995b : 86), dira le narrateur de Un balcon en forêt.

8 Dans Le Rivage des Syrtes, la République d'Orsenna est séparée du territoire ennemi par une frontière invisible. D'un côté, la province des Syrtes "perdue aux confins du Sud " (Gracq, 1989: 558), de l'autre, le Farghestan, séparé du pays voisin par un désert. C'est là où se dresse la frontière de la guerre. À la frontière de la mer des Syrtes se trouve l'Amirauté, une base navale en ruines, dont les fortifications, autrefois puissantes, sont maintenant à l'abandon, «ruine habitée " (Gracq, 1989: 568), évoquant un paysage gothique semblable à ce que nous pouvons trouver dans Au château d'Argol, le premier 
roman de Gracq. Près de cet endroit, il y a la bourgade de Maremma et, au loin, lîle de Vezzano et sa grotte, où apparemment il ne se passe rien. À l'opposé, seuls « les espaces inconnus " existent, comme représentation géographique de la puissance adverse du Farghestan, l'ennemi de toujours et duquel on ne sait presque rien: "Très au-delà, prodigieux d'éloignement derrière cet interdit magique, s'étendaient les espaces inconnus du Farghestan, serrés comme une terre sainte à l'ombre du volcan Tängri » (Gracq, 1989 : 577).

$9 \mathrm{Si}$, comme l'affirme Tadié, « la structure spatiale est capitale, c'est que le franchissement de la frontière, ligne marine immatérielle, déclenche la catastrophe finale » (Tadié, 1990 : 93). Dans cette structure se positionnerait Le Rivage des Syrtes, organisé en fonction de cet « interdit magique » représenté par la frontière et dont la violation constitue l'action principale :

Orsenna et le monde habitable finissaient à cette frontière d'alarme, plus aiguillonnante encore pour mon imagination de tout ce que son tracé comportait de curieusement abstrait ; (...) sans que je voulusse me l'avouer, j'étais prêt à douer de prodiges concrets ce passage périlleux, à m'imaginer une crevasse dans la mer, un signe avertisseur, un passage de la mer Rouge (Gracq, 1989 : 577).

Le sens symbolique du franchissement de cette frontière renvoie le héros à l'imaginaire biblique qui lui promet la «terre sainte ", quoique connotée d'érotisme. Une dynamique de la transgression conjugue sacré et profane à la découverte du volcan Tängri. Ce questionnement rejoint la pensée de Georges Bataille pour qui « tout érotisme est sacré » (Bataille, 1974: 20). La définition reprend la procédure religieuse où l'homme mène la quête de son identité profonde depuis le début des temps, d'une continuité de l'être audelà de sa réalité immédiate. Dans son désir de fusion avec une autre réalité, un sacrifice s'impose : «Essentiellement, le domaine de l'érotisme est le domaine de la violence, le domaine de la violation » (Bataille, $1974: 20)$. La violation permettra le passage d'un état à l'autre, de la réalité immédiate à une réalité inconnue, une violation où paraît s'inscrire également la fascination pour la mort.

11 Dans son projet de transgression, le héros pressentira les risques inhérents à l'acte qui vont lui permettre de comprendre « ce passage périlleux » comme un passage obligé vers les hauteurs de la transcendance, " comme un homme qui se sentirait glisser lentement de l'autre côté du miroir » (Gracq, 1989 : 696).

12 C'est avec le regard pris dans cette ligne imaginaire de l'horizon symbolique entre le réel et le merveilleux, mais aussi entre l'indubitable et le fantastique, que le héros, séduit par l'aventure, choisira la « bonne direction » dans son parcours de vie. « Comme les primitifs qui reconnaissent une vertu active à certaines orientations, je marchais toujours plus alertement vers le sud: un magnétisme secret m'orientait par rapport à la bonne direction» (Gracq, $1989: 611)$.

13 Il convient, donc, de mettre en évidence le fonctionnement du "magnétisme secret » (Gracq, 1989: 611), en tant qu'une quête constante qui se renouvelle et qui se prolonge, une fois que « les lieux magiques » de la liberté et de la pureté originale se rencontrent à une distance infranchissable des mythes. Comme le dira Michel Collot, "l'horizon gracquien est, plus que tout autre, un horizon d'attente, que le guet interroge vainement, et que la quête déplace indéfiniment » (Collot, 1994 : 110).

14 Gracq insistera sur cette idée de renouveau lorsqu'Aldo transgressera la frontière, en essayant de rapprocher ces deux mondes divergents, au lieu de les maintenir à distance. 
J'avais cheminé en absence, fourvoyé dans une campagne de plus en plus morne, loin de la Rumeur essentielle dont la clameur ininterrompue du grand fleuve grondait en cataracte derrière l'horizon. Et maintenant le sentiment inexplicable de la bonne route faisait fleurir autour de moi le désert salé (...) l'horizon tremblé de chaleur s'illuminait du clignement de signaux de reconnaissance - une route royale s'ouvrait sur la mer pavée de rayons comme un tapis de sacre - et, aussi inaccessible à notre sens intime (...) il me semblait que la promesse et la révélation m'étaient faites d'un autre pôle où les chemins confluent au lieu de diverger » (Gracq, 1989 : 735-736).

Le dénouement de cette quête se traduira, dans la fiction de l'auteur, par une catastrophe, pour ainsi dire, déjà pressentie. Le néant qui semble remplir l'horizon s'imposera alors comme l'abîme. On devine la mort, le dernier horizon de l'existence; le texte semble l'annoncer, unissant la mort à l'amour :

Je la sentais [Vanessa] auprès de moi comme le plus profond que pressentent les eaux sauvages, comme au front le vent emportant de ces côtes qu'on dévale les yeux fermés, dans une remise pesante de tout son être, à tombeau ouvert. Je me remettais à elle au milieu de ces solitudes comme à une route dont on pressent qu'elle conduit vers la mer (Gracq, $1989: 623$ ).

C'est cette mer, également synonyme de mort, qu'il faudra traverser afin d'accéder au sacré et à l'amour. Cette mer qui projettera dans la stérilité des terres désertiques son image et sa nature, à la fois fin et commencement :

Je me sentais de connivence avec la pente de ce paysage glissant au dépouillement absolu. Il était fin et commencement. Au-delà de ces étendues de joncs lugubres s'étendaient les sables du désert, plus stériles encore ; et au-delà - pareils à la mort qu'on traverse - derrière une brume de mirage étincelaient les cimes auxquelles je ne pouvais plus refuser un nom (Gracq, 1989 : 611).

Investi d'une portée eschatologique, l'horizon renvoie à la mort, en se présentant comme le seuil du réel, mais aussi de l'espoir ; la frontière peut être synonyme de mort dans la représentation que l'on peut se faire du passage d'un monde à l'autre, différent et meilleur, dans sa stérilité qui l'éloigne du monde référentiel, en le rapprochant du monde sans vie, un « delà - pareil à la mort qu'on traverse » (Gracq, 1989 : 611).

Non sans évoquer Mallarmé, Gracq trouverait dans le néant la vision de l'absolu. Ce serait la raison pour laquelle, dans Le Rivage des Syrtes, le héros ne peut détacher son regard d'un « lointain » (Gracq, 1989 : 558) qui, à son tour, se présente de manière « indéfinie » comme «avenir béant " (Gracq, 1989: 563), associé à sa vision de l'espace, quand Aldo quitte Orsenna.

19 En traversant cette ligne qui sépare les deux pays pendant la guerre, le héros semble revivre « le sentiment suffocant d'une allégresse perdue depuis l'enfance», provoqué par «l'horizon [que] la vision devant nous, se déchirait en gloire» (Gracq, 1989: 733); il éprouve une étrange sensation de liberté et d'une renaissance symbolique, comme un retour aux origines, dans un monde de nouveau purifié, par la vision métaphorique des eaux: «il me semblait que maintenant tout entier j'étais remis - une liberté, une simplicité miraculeuse lavaient le monde; je voyais le matin naître pour la première fois » (Gracq, 1989 : 733-734).

Gracq va ainsi dans le même sens que Mircea Eliade, qui voit l'immersion comme "une réintégration passagère dans l'indistinct, à laquelle se succèdent une nouvelle création, une nouvelle vie ou un homme nouveau » (Eliade, 1992 : 270). Le contact avec l'élément aquatique semble avoir comme finalité l'utilisation «de ce temps-là », donc dans illo tempore, dans lequel eut lieu la création, se révélant comme une répétition symbolique de 
la naissance des mondes ou de "l'homme nouveau » : « Le Farghestan avait dressé devant moi des brisants de rêve, l'au-delà fabuleux d'une mer interdite. (...) La dernière tentation, la tentation sans remède, prenait corps dans ce fantôme saisissable, dans cette proie endormie sous les doigts déjà ouverts » (Gracq, 1989 : 729).

D'Orsenna à cet autre endroit, l'itinéraire d'Aldo se présentera, selon la perspective de Pierre Jourde, comme celui d'un explorateur qui quitte le monde réel pour s'approcher de la bordure d'un continent inconnu (Jourde, 1991 : 26). Dans la figuration hyperbolique de la séparation, la frontière agrandie des eaux nuit aux contacts et en conséquence suscite l'imagination des possibles. Cette mesure des possibles qui, dans Le Rivage des Syrtes prennent forme, à savoir par le biais du volcan Tängri, émergé des eaux et s'assimilant aux contours indéfinis de l'île du Farghestan, tels une représentation phallique. Atteindre cette île - et l'île est cette portion de territoire qui rappelle toujours l'utopie - ne relève pas d'une simple expérience cognitive du regard, cela équivaut surtout à relever les défis d'un grand jeu. «Voir et toucher, (...) se fondre dans cette approche éblouissante, se brûler à cette lumière sortie de la mer» (Gracq, 1989: 745). De là vient la vision de ce « lieu magique », objet fascinant du désir, renvoyant à la fois à l'amour et à la mort. De là vient l'envie de franchir le pas, de dépasser la frontière, de faire «tomber le dernier voile »: "Un charme nous plaquait déjà à cette montagne aimantée. Une attente extraordinaire, illuminée, la certitude qu'allait tomber le dernier voile suspendait ces minutes hagardes » (Gracq, 1989 : 744).

Si la forêt paraît toujours marquer la frontière avec le monde interdit, la mer, cette " entité légendaire douée d'une puissance d'envoûtement ", c'est aussi un lieu séduisant, dont le rôle est de séparer la vision du réel de celle du rêve. «Il me semblait que nous venions de pousser une de ces portes qu'on franchit en rêve » (Gracq, 1989 : 733). Comme les espaces-limites de la représentation de l'absence, toujours silencieux et vides, ils ont tendance à s'imposer, dans la pratique fictionnelle de Gracq, en corrélation avec l'attente et la mort.

Le Rivage des Syrtes «se projette de façon inquiétante, comme le volcan Tängri de son septième chapitre, vers notre avenir » (Vila-Matas, 2007 : 51), affirme Enrique Vila-Matas en anticipant « une vision, plutôt glacée, de l'avenir terrifiant, stérile et chaotique qui attend l'Occident » (Vila-Matas, 2007 : 53). Il souligne ainsi que « Julien Gracq préfigure le paysage moral et littéraire de la civilisation occidentale : un monde voué au vide, marqué par la fin de l'Histoire » (Vila-Matas, 2007 : 51). Les héros de Gracq, désignés dans l'œuvre par « poètes de l'événement » (Gracq, 1989: 775), auront donc pour objectif de surmonter la réalité apparente du monde réel, dans leur parcours, de manière à atteindre le côté obscur : « Passer aussi de l'autre côté, éprouver à la fois la pesée et la résistance » (Gracq, 1989 : 775).

24 D'autres, comme Fabrizio, accompagnent et aident le héros dans le passage de la ligne interdite, en l'incitant à l'aventure. À la fin du roman, tous les autres personnages semblent cesser d'exister; "désormais le décor était planté » (Gracq, 1989: 839). Le dernier chapitre semble se fermer sur le premier, conduisant le narrateur - survivant à la catastrophe - à revivre son aventure : «Lorsque je revis en souvenir les premiers temps de mon séjour dans les Syrtes » (Gracq, 1989: 564).

Arrivé au moment de la transgression, la clôture du roman joue sur une construction circulaire, symbole d'un monde clos, toujours habitée par l'absence. Le livre répète les mêmes motifs tout en orientant les personnages dans sa tentative de dépasser les frontières de la supra-réalité. La construction circulaire renvoie aux endroits 
préférentiels de l'imaginaire de Gracq, «lieux magiques » où se prolonge indéfiniment l'attente du héros, mais aussi celle du lecteur.

Gracq semble ainsi l'affirmer avec Breton: «j'aimerais que ma vie ne laissât après elle d'autre murmure que celui d'une chanson de guetteur, d'une chanson pour tromper l'attente. Indépendamment de ce qui arrive, n'arrive pas, c'est l'attente qui est magnifique » (Breton, 1992 : 697).

\section{BIBLIOGRAPHIE}

BATAILlE, GEORGES (1974). L'Érotisme. Paris : Union Générale d'Édition, « 10/18 ».

BRETON, André (1992). L'Amour fou, in CEuvres complètes, T. II. Paris : Gallimard, Bibliothèque de la Pléiade.

COLLOT, Michel (1994). « Les guetteurs de l'horizon », in Michel Murat (Org.), Julien Gracq 2, Un écrivain moderne. Paris : Revue des Lettres Modernes, pp. 109-126.

ELIADE, Mircea (1992). Tratado de história das religiões. Porto : Edições ASA.

GRACQ, Julien (1989). Le Rivage des Syrtes, in Euvres complètes, T. I. Édition établie par Bernhild Boie. Paris : Gallimard, Bibliothèque de la Pléiade.

GRACQ, Julien (1995a). Lettrines, in Euvres complètes, T. II. Édition établie par Bernhild Boie avec la collaboration de Claude Dourguin. Paris : Gallimard, Bibliothèque de la Pléiade.

GRACQ, Julien (1995 b). Un balcon en forêt, in CEuvres complètes, T. II. Édition établie par Bernhild Boie avec la collaboration de Claude Dourguin. Paris : Gallimard, Bibliothèque de la Pléiade.

ERNST, Gilbert (1997). « Sur Un balcon en forêt », in Jean-Louis Leutrat (dir.). Julien Gracq, Les cahiers de L'herne. Paris : Fayard, pp. 211-222.

Jourde, PIERRE (1991). Géographies imaginaires. Paris : José Corti.

MONIZ, Ana Isabel (2010). Julien Gracq : formas sentidos e mecanismos do imaginário. Lisboa : Edições Cosmos.

RACAULT, Jean-Michel (1995). «Avant-Propos », in J .C. Marimouton, J.M. Racault, (éd.). L'insularité : thématique et représentations. Paris : L'harmattan, PP. 9-13.

TADIÉ, Jean-Yves (1990). Le Roman au XXe siècle. Paris : Belfond, « collection les dossiers belfond ». VILA-MATAS, Enrique (2007). « La froide lumière des Syrtes », Le magazine littéraire nº 465, pp. 51-54. WUNENBURGER, Jean-Jacques (2000). Art mythe et création. Paris : Vrin. 


\section{NOTES}

1. Les citations du roman Rivage desSsyrtes se reporteront à l'édition de la pléiade, vol. i, 1989 ( Julien Gracq, Euvres Complètes, T. I. Paris : Gallimard, Bibliothèque de la Pléiade, Édition établie par Bernhild Boie, de 1989).

2. «Entretien sur Un Balcon en Forêt », entretien radiophonique entre Julien Gracq et Gilbert Ernst, diffusé le 12 juillet 1971 par la station régionale d'Inter-Lorraine-Champagne-Ardennes et publié dans Les Cahiers de L'Herne (Leutrat, 1997 : 211-222).

\section{RÉSUMÉS}

Dans les parcours de l'aventure qui marquent les univers fictionnels de Julien Gracq, tout semble commencer par un déplacement dans l'espace qui s'impose aux héros. Sauf en de rares exceptions, le récit prend la forme d'un voyage annoncé dès l'incipit. Dans Le Rivage des Syrtes, roman élu par le Prix Goncourt et distinction refusée par l'écrivain, la traversée de la mer des Syrtes entreprise par le protagoniste en direction de l'île de Vezzano revêt la même signification symbolique que celle d'un voyage annonciateur d'une découverte. Dans ce projet qui lui fera prendre conscience de l'inconnu, il rompt avec la banalité du quotidien pour, finalement, contempler «la lumière jaillie de la mer». Atteindre cette l'île, ce lieu du désir et de la découverte, c'est le défi posé au héros afin qu'il retrouve son identité à travers un processus symbolique et une pratique de mise en altérité.

On the adventurous paths that mark Julien Gracq's fictional worlds, the heroes are often subjected to a spatial displacement. And this occurs, with few exceptions, throughout the different texts, right from their incipit. In the Prix Goncourt awarded novel Le Rivage des Syrtes for example, - a prize Julien Gracq declined - the protagonist's crossing of the sea of Syrtes to the Island of Vezzano, conveys the same symbolism of a foregoing voyage towards a discovery. This project will allow him to experiment a vision of the forbidden. He breaks away from the everyday life's triviality in order to finally be able to contemplate "la lumière sortie de la mer." The island is the place of desire and discovery. The hero's strife for arriving on the island mimics his process of finding his own identity, by means of the symbolic relation he establishes with the otherness.

\section{INDEX}

Keywords : Gracq (Julien), voyage, island, forbidden, revelation

Mots-clés : Gracq (Julien), voyage, île, interdit, révélation 
AUTEUR

ANA ISABEL MONIZ

Université de Madeira - CEC - Université de Lisboa

anamoniz[at]uma.pt 